\title{
EFEITO DA OXIGENOTERAPIA HIPERBÁRICA: RELATO DE CASO DE PACIENTE COM SÍNDROME HEPATO-PULMONAR EM LISTA DE ESPERA PARA TRANSPLANTE HEPÁTICO
}

\author{
Effect of hyperbaric oxygen therapy: case report of patient with hepato-pulmonary syndrome on \\ waiting list for liver transplantation
}

Flávio KREIMER ${ }^{1}$, Bruno SENA ${ }^{1}$, Álvaro FERRAZ ${ }^{1}$, Josemberg CAMPOS ${ }^{1}$, Luiz Carneiro D'ALBUQUERQUE ${ }^{2}$, Guido C. ARAÚJO Jr. ${ }^{1}$, Edmundo FERRAZ ${ }^{1}$

Trabalho realizado no ${ }^{1}$ Departamento de Cirurgia, Serviço de Cirurgia Geral do Hospital das Clínicas, Universidade Federal de Pernambuco, Recife, PE e 'Serviço de Transplante e Cirurgia do Fígado do Departamento de Gastroenterologia, Faculdade de Medicina da Universidade de São Paulo, SP, Brasil.

DESCRITORES - Oxigenioterapia.

Transplante de fígado.

Correspondência:

Flávio Kreimer

flaviok@nlink.com.br

Fonte de financiamento: não há

Conflito de interesses: não há

Recebido para publicação: 10/09/2009

Aceito para publicação: 23/11/2009

HEADINGS - Oxygen inhalation therapy. Liver transplantation.
RESUMO - Introdução - As doenças hepáticas apresentam índices de morbidade e mortalidade elevados e quando em estágio avançado têm o transplante do fígado como forma de tratamento potencialmente curativo e eficaz, embora este não possa ser oferecido a todos os pacientes. Isso faz com que essas doenças sejam consideradas problema de saúde pública em todo o mundo. Os cuidados clínicos para manter o paciente com condições de esperar e suportar o transplante continua um desafio. Relato do caso - Mulher com 65 anos de idade, procedente do Recife, com diagnóstico de cirrose hepática secundária a vírus $C$ apresentava dispnéia importante aos mínimos esforços tendo $\mathrm{PaO} 2$ de repouso de $60 \mathrm{mmHg}$ e $\mathrm{O} 2$ de $90 \%$, com espirometria normal. Realizou eco-Doppler que evidenciou shunt pulmonar importante. Durante a triagem em lista de transplante (MELD de 16 em agosto de 2006) foi optado pelo início de sessões de oxigenioterapia em câmara hiperbárica a fim de melhorar a sintomatologia respiratória da síndrome hepato-pulmonar. Apresentava melhora substancial da tolerância ao exercício após a terapia hiperbárica, assim como os valores do $\mathrm{PaO} 2$ à gasometria. Realizou 10 sessões de oxigenioterapia em câmara hiperbárica. Realizou transplante hepático em outubro de 2007 e vem em acompanhamento ambulatorial com boa evolução e melhora substancial da dispnéia. Conclusão - Constatou-se melhora da condição hepato-pulmonar após oxigenoterapia hiperbárica. Desta forma, ela surge como mais uma ferramenta para o tratamento das doenças hepáticas, devendo ser realizados outros estudos que avaliem sua utilização clínica.
ABSTRACT - Introduction - Progressive impairment of liver function in hepatic cirrhosis is a major medical problem with limited therapeutic approaches, and liver transplantation is the only effective therapy. Reports of alternatives for liver function improvement on patients awaiting liver transplantation may include hyperbaric oxygen therapy. Case report - A 65-year old woman had diagnosis of hepatitis C and liver cirrhosis during treatment for breast cancer. In addition to liver failure and a MELD of 16, the patient presented hypoxemia with a $\mathrm{PaO} 2$ of $60 \mathrm{mmHg}$, and further evaluation with eco-Doppler revealed a hepatopulmonary syndrome. Clinical improvement was attempted with hyperbaric oxygeniotherapy, and substantial clinical and laboratorial improvement was acheived after 10 sessions, with significant improvement on cyanosis and arterial gas values. Conclusion - Oxygen therapy was effective in the improvement of dyspnea and hypoxemia secondary to hepatopulmonary syndrome in a patient with liver cirrhosis indicated for liver transplantation. The positive results of this treatment suggest that hyperbaric oxygen therapy may help on improvement of symptoms of hepatopulmonary syndrome.

\section{INTRODUÇÃO}

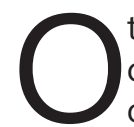
transplante de fígado é procedimento cirúrgico complexo da cirurgia moderna; nenhum outro interfere com tantas funções do organismo ${ }^{5}$. O fígado desempenha inúmeras funções metabólicas além do clareamento sanguíneo de bactérias por meio da ação fagocitária das 
células de Kupffer ${ }^{3}$. Estudos mostraram que quando necessário sofrem a ação de um estímulo regenerativo e 95\% das células desencadeiam sua reprodução mitótica, enquanto mantêm suas funções metabólicas ${ }^{4}$.

O tempo decorrido entre o agravamento da doença hepática e a indicação de transplante pode exercer impacto sobre a morbidade e a mortalidade pós-transplante. A condição clínica até o momento da operação, pode inclusive contra-indicá-la. Pacientes transplantados antes do desenvolvimento de complicações em múltiplos sistemas em estágio avançado têm intervalo de tempo de sobrevida grande, com qualidade de vida; todavia os debilitados, com falência de múltiplos órgãos antes do transplante podem vir a ter de $20 \%$ a $30 \%$ apenas de sucesso e, frequentemente, necessitam semanas ou mesmo meses de hospitalização pós-operatória, o que representa altos custos em sistema de saúde de poucos recursos ${ }^{2}$.

Em hepatologia, estudos sobre o emprego da oxigenoterapia hiperbárica se iniciaram em 1968, quando foi empregada para conservação de enxertos hepáticos ${ }^{1}$, mas foi em 2001 que pesquisadores simulando a embolização pré-operatória da veia porta pela ligadura de seu ramo direito, comprovaram aumento significante dos níveis séricos do fator de crescimento de hepatócitos, aumento da proliferação celular e hipertrofia compensatória nos segmentos hepáticos não ligados, em ratos submetidos à oxigenoterapia hiperbárica6 .

Os autores apresentam um caso de um portador de doença hepática em estágio avançado, com indicação de transplante, com evolução favorável onde foi utilizada oxigenoterapia hiperbárica antes do transplante.

\section{RELATO DO CASO}

Mulher com 65 anos de idade, natural e procedente de Recife, durante exames de rotina foi identificado fígado cirrótico ao exame de imagem; encaminhada ao serviço de Hepatologia do HC-UFPE, iniciou propedêutica e diagnóstico da cirrose hepática secundária a vírus $C$.

Foi decidido tratamento com ribavirina associado a interferon (3.000.0003/3dias) em 1994, porémapresentou trombocitopenia importante com sangramentos gengivais repetidos o que trazia risco impeditivo para o uso do interferon (suspenso em fevereiro de 1995). Foi solicitado mielograma que mostrou hiperplasia medular reacional. Optou-se então por esplenectomia afim de aumentar os níveis plaquetários, tendo em vista reiniciar o uso do interferon. O procedimento foi feito em agosto de 1996 e transcorreu sem intercorrências. Evoluiu com tosse seca e eventuais episódios de dispnéia que se tornaram cada vez mais frequentes e graves associados a quadros de encefalopatia hepática.

Ela evoluiu com dispnéia importante aos mínimos esforços tendo um $\mathrm{PaO} 2$ de repouso de
$60 \mathrm{mmHg}$ e saturação de 89/90\% com espirometria normal. Realizou-se eco-Doppler que evidenciou shunt pulmonar importante. A paciente que já estava em lista de transplante (MELD de 16 em agosto de 2006) foi incluída em protocolo e iniciadas sessões de oxigenioterapia em câmara hiperbárica a fim de se tentar melhorar a sintomatologia respiratória da síndrome hépato-pulmonar.

Ela apresentava melhora substancial da tolerância ao exercício após a terapia hiperbárica, assim como os valores do $\mathrm{PaO}^{2}$ à gasometria. Realizou 10 sessões no total.

Foi submetida à transplante hepático em outubro de 2007 e vem em acompanhamento ambulatorial com boa evolução e melhora substancial da dispnéia.

No exame físico durante todo o período de avaliação havia cianose de extremidades. Houve melhora após a terapia hiperbárica.

\section{DISCUSSÃO}

Os resultados do presente relato estimulam a realização de estudos utilizando a oxigenoterapia hiperbárica para portadores de doenças hepáticas em estágio avançado, particularmente portadores da síndrome hépato-pulmonar com indicação de transplante hepático, como forma de melhorar seu estado respiratório e geral, dando-lhes maiores chances a serem submetidos ao transplante e em melhores condições.

Seria a oxigenioterapia em câmara hiperbárica tratamento adjuvante na sintomatologia respiratória dos pacientes com síndrome hépato-pulmonar enquanto aguardam o transplante hepático?

Não encontrou-se na literatura dados relacionados a esta conduta. Na verdade a paciente fazia parte de protocolo de pesquisa do serviço que utilizava a o oxigenoterapia hiperbárica em pacientes selecionados graves e com poucas perspectivas de alcançar 0 transplante hepático.

O intuito deste relato é o registro de um achado incidental ocorrido em outro estudo em fase de conclusão. Deve suscitar questionamentos e estudos controlados que aprofundem o conhecimento e possam responder o papel da oxigenoterapia hiperbárica em hepatopatas crônicos e aos submetidos à ressecções hepáticas, tromboses arteriais pós-transplante e síndrome hépatopulmonar em lista de transplante hepático.

\section{CONCLUSÃO}

Constatou-se melhora da condição hépatopulmonar após oxigenoterapia hiperbárica. Desta forma ela pode surgir como mais uma ferramenta para o tratamento das doenças hepáticas em estágio avançado. Outros estudos se fazem necessários para confirmar esta observação. 


\section{REFERÊNCIAS}

1. Brettscneider L, Daloze PM, Huguet C, Porter KA, Groth CG, Hutchison DE, Starrlz TE. The use of combined preservation techniques for extended storage of orthotopic liver homografts. Surg Gynecol Obstet 1968; 126:263-26.

2. Carithers JR. RL. Liver transplantation. AASLD Practice Guidelines. Liver Transplantation. 2000;6(1):122-35.

3. Fausto N. Liver regeneration. J Hepatology. 2000;32:19-31.
4. Michalopolus GK, Defrances MC. Liver regeneration. Science. 1997;276:60-6.

5. Mies S. Transplante de fígado. Rev Ass Med Brasil. 1998;44(2):12734.

6. Uwagawa T, Unemura $Y$, Yamazaki $Y$. Hyperbaric oxygenation alter portal vein emobilization for regeneration of the predicted remnant liver. J Surg Res. 2001;100(1):63-8. 